msh-mss Mathématiques et sciences humaines

183 | Automne 2008

Hommage à Georges-Th. Guilbaud

\title{
Publications de G.-Th. Guilbaud
}

G.-Th. Guilbaud publications

\section{(2) OpenEdition \\ 12 Journals}

Édition électronique

URL : http://journals.openedition.org/msh/10753

DOI : $10.4000 / \mathrm{msh} .10753$

ISSN : $1950-6821$

Éditeur

Centre d'analyse et de mathématique sociales de l'EHESS

Édition imprimée

Date de publication : 14 décembre 2008

Pagination : 17-23

ISSN : 0987-6936

\section{Référence électronique}

"Publications de G.-Th. Guilbaud », Mathématiques et sciences humaines [En ligne], 183 | Automne 2008, mis en ligne le 15 décembre 2008, consulté le 23 juillet 2020. URL : http:// journals.openedition.org/msh/10753; DOI : https://doi.org/10.4000/msh.10753 


\section{PUBLICATIONS DE G.-Th. GUILBAUD}

\section{OUVRAGES}

1945 (avec R. Daval) : Le raisonnement mathématique, Paris, Presses Universitaires de France, 152 pages, (Nouvelle encyclopédie philosophique).

1947 (avec Y. Mainguy) : Les différents comportements du consommateur et leur détermination statistique, Paris, ISEA, IV, 63 pages.

1949 : Les mathématiques de l'économiste : leçons sur les équations fonctionnelles, Cahiers de l'I.S.E.A., série E, $\mathrm{n}^{\circ}$ 1, Paris, 229 pages.

1950 (avec R. Daval) : La méthode statistique, fasc. 1, Paris, Presses Universitaires de France.

1951 (avec R. Daval) : La méthode statistique, fasc. 2, Paris, Presses Universitaires de France.

1954(1) : La cybernétique, Paris, Presses Universitaires de France, 128 pages, (Que sais-je ?, 638).

Édition en japonais, 1956, Tokyo, Hakusuisha, Que sais-je ?, 197.

Édition en espagnol, 1956, Barcelone, Vergara, Colección « Nuevos horizontes », 6.

Édition en anglais, 1959, Londres, Heinemann, Contemporary sciences series.

Édition en hébreux, 1960, Tel-Aviv.

1954(2) : Leçons sur les éléments principaux de la théorie mathématique des jeux, Paris, CNRS, 126 pages.

1968(1) : Statistique des chroniques, Paris, Dunod, 127 pages, (monographies de recherche opérationnelle, 8).

1968(2) : Eléments de la théorie mathématique des jeux, Paris, Dunod, 143 pages, (monographies de la recherche opérationnelle, 9).

1963 : Mathématiques, collection «Thémis », Paris, Presses Universitaires de France, 2e édition, 1966, 243 pages.

1985 : Leçons d'à peu près, Paris, Christian Bourgois, 235 pages.

1993 : Mathématique sociale, entretien avec G.-Th. Guilbaud. DVD série « Savoir et Mémoire » $n^{\circ} 4$, Paris, AREHESS, [consultable sur le site : http//www.ehess.fr]. 


\section{ARTICLES}

1946(1) (avec H. Guitton) : «Déterminisme et marché », Revue d'économie politique $n^{\circ} 4,409-435$.

1946(2) : «Une méthode d'analyse sommaire de la structure démographique », Économie et humanisme, $\mathrm{n}^{\circ} 27,515-525$.

1947(1) (avec H. Guitton) : " Déterminisme et marché », Revue d'économie politique, 332-335, [suite de 1946(1)].

1947(2) : Science et technique, rapport aux XXIV Journées universitaires, 24-51.

1948(1) : "Sur les méthodes d'étude des budgets de ménage », Cahiers de l'Institut de sciences économiques appliquées, série B, annexe 4, Paris, 46-63.

1948(2) (avec S. Dupin) : «Études sur la consommation de pain d'après les budgets de ménage », Bulletin de l'Institut national d'hygiène, tome $3, \mathrm{n}^{\circ} 3$, juillet-septembre, 408-410.

1948(3) : «La correction des erreurs accidentelles dans les comptabilités approchées », Cahiers de l'Institut de sciences économiques appliquées, série D, n ${ }^{\circ}$, Paris, 141-169.

1949(1) (avec J. Marczewski) : «Essai d'analyse graphique d'une comptabilité nationale », Économie appliquée, 138-169.

1949(2) : «La théorie des jeux, contribution critique à la théorie de la valeur», Economie appliquée, 275-319. Traduction en anglais dans International Economic Papers, $\mathrm{n}^{\circ}$ 1, London, McMillan, 1951, 37-65. [Reproduit dans Mathématiques et action politique : études d'histoire et de philosophie des mathématiques sociales, sous la direction de Th. Martin, INED, Paris 2000].

1949(3) : « Note sur l'économétrie des fluctuations », Économie Appliquée, 429-495.

1950(1) : «Quelques applications de la théorie mathématique des réseaux », communication au Congrès européen de la Société internationale d'économétrie, Colmar, 12 septembre 1949, résumé dans Econometrica, vol. 18, n 1, 73-74.

1950(2) : «Contributions à la théorie des réseaux : situations de la théorie des réseaux de la comptabilité », Économie appliquée, 159-164.

1950(3) : «Calculs symboliques et calculs financiers », (Congrès d'économétrie de Varèse), Économie appliquée, 353-394. Résumé dans Econometrica 19, 1951, 192-194.

1950(4) : «Divagations cybernétiques », revue Esprit, Paris, septembre 281-295.

1951(1) : «L'étude statistique des oscillations économiques », Cahiers du séminaire d'économétrie, Paris, CNRS, 58-93.

1951(2) (en collaboration) : «Le revenu national français de 1780 à nos jours », rapport à la réunion de Royaumont de l'International Association for Research in Income and Wealth (IARIW), Income and Wealth, series 3, Cambridge, Bowes, 1953, 46-66 et « La croissance du revenu national français depuis $1970 »$, Cahiers de l'Institut de sciences économiques appliquées, série $\mathrm{D}, \mathrm{n}^{\circ}$ 7, Paris, 1952, 13-52.

1951(3) : «En marge de Schumpeter : quelques espérances mathématiques », Économie appliquée $\mathrm{n}^{\circ} 2,242-269$.

1952(1) (avec W. Jaffé) : «La correspondance complète de Cournot et Walras (18731875) », établie et annotée, Économie appliquée, n ${ }^{\circ}$ 1, 5-33. 
1952(2) : «Le problème du partage : matériaux pour une enquête sur les algèbres et les arithmétiques de la répartition », Économie appliquée, 93-137.

1952(3) : "Les théories de l'intérêt général et le problème logique de l'agrégation », Economie appliquée, 501-584. Traduction en anglais des pages 507 à 572 dans Reader on mathematical social sciences, UNESCO, Paris, 1966. Traduction complète : "Theories of the general interest and the logical problem of aggregation », Journal électronique d'Histoire de la Probabilité et de la Statistique (www.jehps.net), vol. 4, $\mathrm{n}^{\circ} 1,2008$.

1953(1) : «Note sur l'étude statistique des croissances et de leurs irrégularités », présentée à la troisième conférence de l'International Association for Research in Income and Wealth (Castelgandolfo), 1-28.

1953(2) : «Pilotes, stratèges et joueurs », conférence faite à un colloque organisé à la Sorbonne par la Maison des Sciences, Paris, le 24 mars 1953, publiée dans Structure et évolution des techniques, $\mathrm{n}^{\circ}$ spécial de Cybernétique, $5^{\mathrm{e}}$ année, $\mathrm{n}^{\circ} 35-36$, juillet $1953-$ janvier 1954, Paris. [Réédité et mis à jour dans la Revue internationale de systémique, vol. 2, n 3, 1988, 321-344].

1953(3) : Influence du changement d'échelle sur les propriétés de groupes sociaux, Rapport sur le programme du Conseil international des sciences sociales (assemblée générale, Paris, Unesco, novembre 1953, document WP 2).

1953(4) : « Sur une difficulté de la théorie du risque », Le risque, colloque international du CNRS n XL, Econométrie, Paris, CNRS 19-28.

1954(1) : «Stratégies et décisions économiques, vers une science de la conduite de l'action humaine », conférences faites à l'Institut français du Royaume-Uni (Londres et Edimbourg) en janvier 1953, publiées sous le titre «Stratégies et décisions », La vie intellectuelle, $\mathrm{n}^{\circ}$ d'août-septembre 1954, 36-57.

1954(2) : «Le statisticien est-il un joueur ?» (théories de la décision statistique), conférence à la Société de statistique de Paris, 20 janvier 1954, in Journal de la société de statistique de Paris, 1956.

1954(3) : «Les méthodes de travail dans l'étude des vocabulaires techniques », exposé au Congrès de l'association française pour l'avancement des sciences, Poitiers, 19 juillet 1954, $18^{\mathrm{e}}$ section économie et sociologie.

1955(1) : «Le choix des horaires sur un réseau de transports en commun », exposé fait en janvier 1955 au séminaire de recherche opérationnelle, bulletin $n^{\circ} 2$, publié dans Revue de statistique appliquée.

1955(2) : «La théorie des jeux », rapport au congrès des économistes de langue française, Paris, 24 mai 1954, publié dans Revue d'économie politique.

1955(3) : «La recherche opérationnelle en France », Actes du congrès de l'Institut international de statistique, $29^{\mathrm{e}}$ session, Rio de Janeiro, 395-398.

1956(1) : «La recherche opérationnelle et ses applications », Revue de statistique appliquée (4-3), 7-20.

1956(2) : «Pour une étude de la recherche opérationnelle », Revue française de recherche opérationnelle, vol. $\mathrm{I}, \mathrm{n}^{\circ} 1,3-12$.

1956(3) : "Circulation aléatoire dans un réseau », colloque international du CNRS $\mathrm{n}^{\circ}$ LXII, Les modèles dynamiques, 117-125. 
1956(4) : Préface de l'édition française de La stratégie dans les actions humaines : les affaires, la guerre, les jeux, par J.D. Williams, Paris, Dunod.

1957(1) : «Introduction à la théorie des décisions statistiques par l'analyse d'un jeu séquentiel », Cahiers de l'Association pour le développement des techniques de marchés, $\mathrm{n}^{\circ} 2$, 41-52.

1957(2) : «Programmes dynamiques et programmes linéaires. Note sur un modèle de Richard Bellman », Cahiers du Bureau universitaire de recherche opérationnelle, cahier $n^{\circ}$ 2, Paris, I.S.U.P., 37-41.

1958 : «Approximation entropique et plans économiques de rangement », colloque CNRS Calcul des probabilités, Paris, 143-147.

1959(1) : «Les problèmes de la statistique », chapitre VI du Traité de sociologie, sous la direction de G. Gurvitch, Paris, Presses Universitaires de France. Réédité dans mathématiques et Sciences humaines 135, automne 1996, 33-50. Traduction espagnole in Empiria, n 9, 2005, Madrid, U.N.E.D., 211-240.

1959(2) : Préface de l'édition française de Théorie des jeux et programmation linéaire par S. Vajda, (traduit et adapté par J. Bouzitat), Paris, Dunod, 2 édition 1968.

1960 : Préface de Les choix économiques : décisions séquentielles et simulation par P. Rosenstiehl et A. Ghouila-Houri, Paris, Dunod.

1961 : «Faut-il jouer au plus fin ?», Notes sur l'histoire de la théorie de jeux, in colloque CNRS La Décision, 25-30 mai 1959, Paris, 171-182.

1962(1) : «Pascal et la mathématique » (pour le tricentenaire de la mort de Pascal), La table ronde 171, Paris, 104-122.

1962(2): «Petite introduction à la combinatoire», Revue française de recherche opérationnelle 24, 243-260.

1962(3) : «Pour qu'on lise Pascal », Revue française de recherche opérationnelle 24, 195-198.

1963(1) : «Un exercice sur les permutations », Mathématiques et Sciences humaines 2, 37-43.

1963(2) : «Un exercice sur les permutations » (suite), Mathématiques et Sciences humaines 3, 43-51.

1963(3) : (avec P. Rosenstiehl), « Analyse algébrique d'un scrutin », Mathématiques et Sciences humaines 4, 9-33. (Réédité, revu et augmenté dans Ordres totaux finis, travaux du séminaire sur les ordres totaux finis, Aix-en-Provence, juillet 1967, Paris, MoutonGauthier-Villars, 1971, collection Mathématiques et Sciences humaines, $\mathrm{n}^{\circ}$ 12, 70-100). 1963(4) : «Un exercice sur les permutations » ( $2^{\mathrm{e}}$ suite), Mathématiques et Sciences humaines 4, 47-48.

1964(1) : (avec M. Eytan), « Présentation de quelques monoïdes finis », Mathématiques et Sciences humaines 7, 3-10.

1964(2) : «Une distribution singulière », Mathématiques et Sciences humaines 8, p. 35.

1964(3) : Analyses de H. Weyl, «Symétrie», Paris, Flammarion, et H. Steinhaus, «Mathématiques en instantanés », Paris, Flammarion, dans Mathématiques et Sciences humaines 8, 41-42.

1964(4) : «La règle des partis et la ruine des joueurs », Mathématiques et Sciences humaines 9, 1964, 3-13. 
1964(5) : (J. Gardelle, avec la collaboration de G.-Th. Guilbaud), «Cadences », Mathématique et Sciences humaines 9, 31-38.

1964(6) : «Avant-propos » à Symétrie et mathématique moderne de Hermann Weyl, Paris, Flammarion, 5-7.

1964(7): Dictionnaire des jeux, sous la direction de René Alleau, Paris, Tchou. Articles :

«Cartes (possibilités et probabilités de distribution), 102-103.

« Dés (possibilités et probabilités de combinaisons des dés), 159-161.

« Jeux (théorie des), 254-270.

«Roulette », 448-452.

1965 : «Deux fois treize ou une théorie du schisme», Mathématiques et Sciences humaines 12, 31-35. [Réédité avec une conclusion supplémentaire dans Archives des sciences sociales des religions, vol. 45, $\left.\mathrm{n}^{\circ} 2,1978,181-187\right]$.

1966(1) : «Exercices de calcul pour préparer à l'usage du Khi deux », Mathématiques et Sciences humaines 14, 31-40.

1966(2) : «Un exercice de logique formelle », Mathématiques et Sciences humaines, $15,29-32$.

1966(3) : «Un exercice de calcul logique chez les indiens des prairies », Mathématiques et Sciences humaines 15, 33-35.

1966(4) : «Pour le 200 e anniversaire de la mort de G.W. Leibniz: un problème leibnizien : les partages de nombres », Mathématiques et Sciences humaines, 17, 13-36.

1966(5) : «À propos des groupes et de leurs axiomatiques », Mathématiques et Sciences humaines 17, 62-63.

1966(6) : «Theories of the general interest », Readings in mathematical social sciences Lazarsfeld (ed.), Chicago, S.R.A., 262-307.

1967(1) : (sous le pseudonyme de C. Lawoll), «Traverser le miroir », Mathématiques et Sciences humaines 20, 29-32.

1967(2) : «Bribes méthodologiques (1): pouvoir des simplexes », Mathématiques et Sciences humaines 20, 33-46.

1968(1): «L'algorithme de l'élagueur», Mathématiques et Sciences humaines 21, $19-28$.

1968(2): «Les partitions et la notation simpliciale», Mathématiques et Sciences humaines 22, 27-31.

1968(3) : «Quelques réflexions mathématiques sur les équilibres économiques » (leçons faites au Centro Matematico Internazionale, l'Aquila, 1965), Revue d'Informatique et de Recherche Opérationnelle 8, 5-39.

1969(1) : «Objets simpliciaux (à suivre) », Mathématiques et Sciences humaines 26, 17-31.

1969(2) : «Esquisses mésologiques », Mathématiques et Sciences humaines 28, 7-25.

1970(1): «Préférences stochastiques », Mathématiques et Sciences humaines 32, 45-56.

1970(2) : «Système parental et matrimonial du Nord Ambrym », Journal de la Société des Océanistes 26, tome XXVI, Paris, Musée de l'Homme, 9-32. [Reproduit dans le présent numéro, p. 72-96]. 
1971 : Avant-propos au numéro spécial : «Quelques aspects de la formalisation en linguistique : $1 »$, Mathématiques et Sciences humaines 34, p. 5.

1973(1) : «Qu'est-ce qu'une permutation ? », Mathématiques et Sciences humaines 42, $5-16$.

1973(2) : «Algèbre de Boole », Combinatoire, graphes et algèbres, Centre de Mathématique Sociale (avec le concours de l'UNESCO), Paris, Gauthier-Villars, 87-102.

1973(3) : «Objets simpliciaux », Combinatoire, graphes et algèbres, Centre de Mathématique Sociale (avec le concours de l'UNESCO), Paris, Gauthier-Villars, 103-122.

1973(4) : «Les langages d'espace », Les applications nouvelles des mathématiques et l'enseignement secondaire, $3^{\mathrm{e}}$ séminaire organisé par la commission internationale de l'enseignement mathématique, Echternach (Grand Duché du Luxembourg), impr. Victor, Esch-sur-Alzette (Luxembourg), 1975, 29-40.

1974(1) : «À propos de l'estimation du nombre des coins », Bulletin de la Société française de numismatique 7, 29 année, 625-634. [Reproduit dans le présent numéro, p. 97-106].

1974(2) : «Permutations tropicales », Colloque Permutations, Paris, juillet 1972. Actes publiés sous le titre Permutations, Paris, Gauthier-Villars, 271-276.

1974(3): «Les discours d'espace et leurs formes mathématiques », symposium de l'association de psychologie scientifique de langue française, Bruxelles, 1972. Actes publiés sous le titre De l'espace corporel à l'espace écologique, Paris, Presses Universitaires de France, 297-324.

1974(4) : «Espaces et mathématiques », colloque «Sémiotique de l'espace », Paris, Institut de l'environnement, mai 1972. Actes publiés sous le titre: Notes méthodologiques en architecture et en urbanisme 3/4, Paris, Institut de l'environnement, 283-293.

1974(5) : «Mathématiques et à peu près », Hasardons-nous, Paris, brochure A.P.M.E.P., ${ }^{\circ} 17,7-29$.

1975(1) : (sous le pseudonyme Thomas Reginal), «Un petit exercice de statistique linguistique », L'Echo des Messaches ${ }^{\circ}$ 0, Paris, novembre 1974, (n.p.).

1975(2) : (non signé), «Au sujet d'un petit exercice... », L'Echo des Messaches 1, Paris, (n.p.).

1975(3) : (non signé), « Lettre à l'éditeur », L'Echo des Messaches 2, Paris, (n.p.).

1975(4) : (non signé), «Variétés. Small samples (sur une poésie de Udny Yule) », L'Echo des Messaches 4, Paris, (n.p.)

1976(1): Avant-propos au numéro spécial: «Une question de praxéologie mathématique », Mathématiques et Sciences humaines 54, 5-7.

1976(2): "Mathématique et approximation », $3^{\text {ème }}$ congrès international sur l'enseignement des mathématiques, Karlsruhe, 19 août 1976, 1-27.

1977: «Les Teilnenner de la Kettenbruche ou : Pareto et Zipf chez Archimède », L'Echo des Messaches 6, Paris, 9-11.

1978 : «Continu expérimental et continu mathématique », Mathématiques et Sciences humaines 62 ( $\mathrm{n}^{\circ}$ spécial « Modélisation des préférences et quasi-ordres »), 11-33. 
1979: «Note sur les comptabilités markoviennes », Mathématiques et Sciences humaines, 66, 99-112.

1980(1) : «Relations entre deux coefficients de corrélation de rangs », Mathématiques et Sciences humaines 72, 45-59.

1980(2) : «Statistique et philologie », $3^{\text {e }}$ colloquio Centro del lessico intelletuale europeo, Roma, Edizioni dell'Ateneo, 11-35.

1980(3) : «Zipf et les fréquences », Mots, Presses de la F.N.S.P., n 1, 97-126.

1980(4): «Les décimales de $\Pi$ et la statistique », Le petit Archimède 64-65, [supplément, $\mathrm{n}^{\circ}$ spécial П, 64-65], 204-221.

1980(5) : (signé Théophraste), « Le tombeau d'Archimède », Le petit Archimède 64-65, [supplément, $\mathrm{n}^{\circ}$ spécial $\left.\Pi, 64-65\right], 230-231$.

1981 : (attribué à, signé Zanzara), «Histoire de crânes ou gare aux arrondis », L'Echo des Messaches 11, Paris, 13-14.

1982 : «Fréquences et probabilités », Analisi delle frequenze, centro del lessico intelletuale europeo, Roma, Edizioni dell'Ateneo, 39-61. Repris dans Informatique et Sciences humaines (Université Paris-Sorbonne), n 61, 1984, 49-67.

1988 : «Envoi », L'à-peu-près, aspects anciens et modernes de l'approximation, Paris, éditions de l'EHESS, p. 255.

1991 : «In Memoriam : P. Costabel », Annuaire des anciens élèves de l'ENS 1991.

1993 : « Te Deum Laudamus », Liturgie 87, 323-338.

1998 : «Fantaisie philologique », Des mots en liberté : mélange Maurice Tournier, ENS (coll. Hommages), vol. 1, 171-175.

2003 : «La ténébreuse affaire de Corcelles », Arithmétique politique dans la France du XVIII siècle, Th. Martin (éd.), Paris, INED, 249-258.

\section{COURS EN FACULTE}

1955 : Les mathématiques de l'économiste, cours 1954-1955, Institut de préparation scientifique aux études économiques.

1961 : Mathématiques : licence $1^{\text {ère }}$ année, sous la direction de G.-Th. Guilbaud, cours rédigés par J.-L. Petit, Les cours de droit, 339 pages.

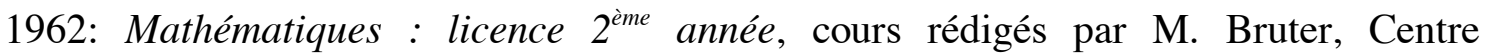
d'économétrie de la Faculté de droit et de sciences économiques de Paris.

1963: Mathématiques : licence en sciences économiques $3^{\text {ème }}$ année, cours rédigés par G. Romier et al., Librairie Dey, 262 pages. 